\title{
BUILDING DAMAGE EXTRACTION TRIGGERED BY EARTHQUAKE USING THE UAV IMAGERY
}

\author{
Shaodan $\mathrm{Li}^{1}$, Hong Tang $2,3, *$ \\ ${ }^{1}$ School of Resource and Environmental Science, Hebei Normal University, 050024, Shijiazhuang, China - lishaodan@ @ebtu.edu.cn \\ ${ }^{2}$ State Key Laboratory of Earth Surface Processes and Resource Ecology, Beijing Normal University, 100875, Beijing, China \\ ${ }^{3}$ Key Laboratory of Environment Change and Natural Disaster, Ministry of Education, Beijing Normal University, 100875, Beijing, \\ China - hongtang@bnu.edu.cn
}

Commission III, ICWG III/Iva

KEY WORDS: Building Damage Information, Earthquake Disasters, Image Fusion, Point Clouds, UAV Imagery

\begin{abstract}
:
When extracting building damage information, we can only determine whether the building is collapsed using the post-earthquake satellite images. Even the satellite images have the sub-meter resolution, the identification of slightly damaged buildings is still a challenge. As the complementary data to satellite images, the UAV images have unique advantages, such as stronger flexibility and higher resolution. In this paper, according to the spectral feature of UAV images and the morphological feature of the reconstructed point clouds, the building damage was classified into four levels: basically intact buildings, slightly damaged buildings, partially collapsed buildings and totally collapsed buildings, and give the rules of damage grades. In particular, the slightly damaged buildings are determined using the detected roof-holes. In order to verify the approach, we conduct experimental simulations in the cases of Wenchuan and Ya'an earthquakes. By analyzing the post-earthquake UAV images of the two earthquakes, the building damage was classified into four levels, and the quantitative statistics of the damaged buildings is given in the experiments.
\end{abstract}

\section{INTRODUTION}

Building damage triggered by earthquakes is a major reason of mortality. The amount and grade of building damage are essential information for rescue and recovery, and it stresses the importance of a rapid and reliable detection of damaged areas. With the rapid development of spatial information technology, remote sensing imagery has become very important data to identify and assess the damaged buildings in hard-hit areas. Recently, many studies using satellite and aerial images to detect earthquake-induced building damage have been completed because of the high spatial resolution and relatively low cost (Dong et al., 2013, Gamba et al., 2007).

For the masonry and reinforced buildings, the European Macroseismic Scale 1998 (EMS98) gives five damage grades, which include slight damage, moderate damage, heavy damage, very heavy damage, and destruction, and each grade gives a clear descript of building damage (Grunthal, 1998). In 2009, China Earthquake Administration (CEA) also put forward the corresponding damage classification standards (GB/T 243352009). Similarly, the building damage is also classified into five grades.

As is known, the building damage grade is measured according to field survey. However, remote sensing imagery provides the central projection of the buildings from the view of the hawkeye, i.e., roof information, but the walls of buildings are difficult to detect from the satellite images. For example, if there are some cracks in the walls, it can not be detected using the satellite images. Therefore, the building damage classification based on remote sensing imagery is difficult to reach the accuracy with field survey. On the other hand, the existing building damage grade did not meet the requirement. For example, the application of LiDAR data makes it possible to detect the specific damage type for individual damaged buildings, such as incline plane, heap of debris, pancake collapsed with different stories (Schweier et al., 2006). Thus it is difficult to construct an explicit correspondence between the building damage grades (e.g., from EMS98) and their appearance in remote sensing imagery.

In order to achieve satisfactory result in building damage detection, varying damage grading schemes are defined according to the type of the remote sensing imagery used. Since LiDAR data can detect many specific damage types for damaged buildings, only the satellite images are discussed here. According to different methods and different resolutions, the damage category can be divided into three cases: (1) two grades. Saito et al visually interpreted collapsed buildings using preand post-seismic IKONOS images of the 2001 Gujarat earthquake. Gusella et al proposed an object-based method to quantify collapsed buildings using QuickBird images taken before and after Bam earthquake, and an overall accuracy of $70.5 \%$ was obtained by this method. Li et al presented a method using both spectral and spatial information of QuickBird imagery to detect damaged buildings in the 2008 Wenchuan earthquake. The combination of spectral and spatial features produced a better outcome compared with using spectral information alone. (2) Three grades. Liu et al proposed an automatic method to detect undamaged, slightly damaged, and collapsed buildings using the regional structure and texture information from post-event IKONOS images of Bhuj earthquake. Gamba et al detected undamaged, partly collapsed, 

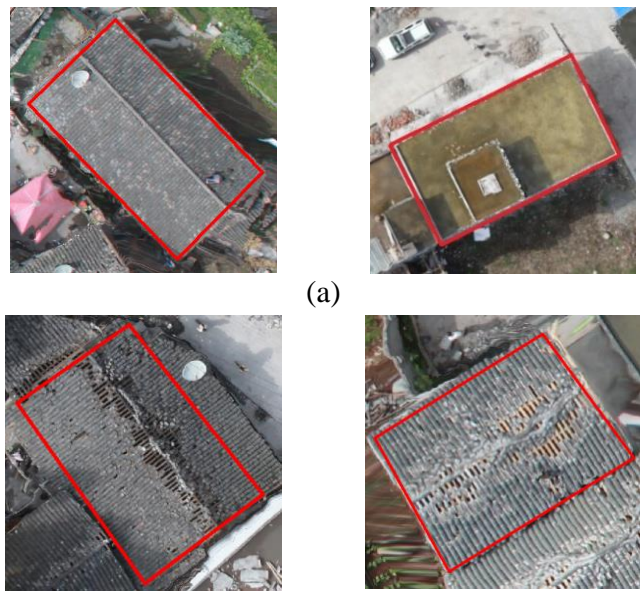

(a)

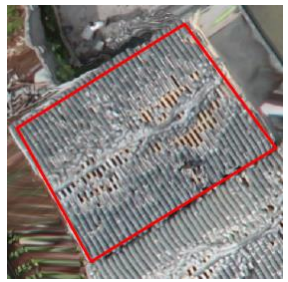

(b)
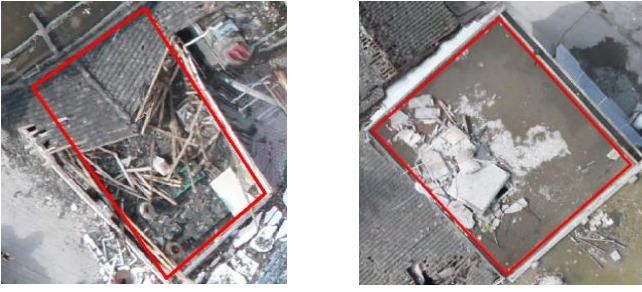

(c)
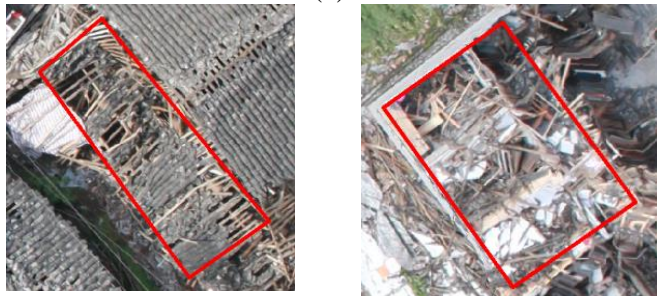

(d)

Figure 1. UAV images for four types of building damage classification: (a) basically intact buildings; (b) slightly damage buildings; (c) partially collapsed buildings; (d) totally collapsed buildings.

and totally collapsed buildings by comparing the normalized difference vegetation index (NDVI) and linear segments of buildings in pre- and post-event images of Tsunami earthquake. Tong et al takes DSMs created from pre- and post-seismic IKONOS stereo image pairs to detect not-collapsed, halfcollapsed, and totally collapsed buildings of Wenchuan earthquake from both building and block levels. (3) More than three grades. Chesnel et al proposed a method based on analysis of building roofs in pre- and post-event QuickBird images to detect the damaged buildings, and they used EMS98 damage scale as a reference scale. Since it is difficult to detect Grade 1 and Grade 2, only intact, Grade 3, Grade 4, and Grade 5 are retained. Iwasaki et al detected mid-story collapsed buildings based on their height change estimated from shadow lengths in pre- and post-event QuickBird images, and the building damage was classified into four levels: totally collapsed (Grade 5), partially collapsed (Grade 4), surrounded by debris (Grade 3), and combined Grade 1 and 2, the EMS98 damage scale is taken as a reference scale. Yamazaki et al detected the debris areas using a combination of edge texture and multi-spectral gray tone, and none $(<25 \%)$, light $(25 \% \sim 50 \%)$, moderate $(>50 \%)$, and totally collapsed buildings are classified according to the ratio of the debris areas.
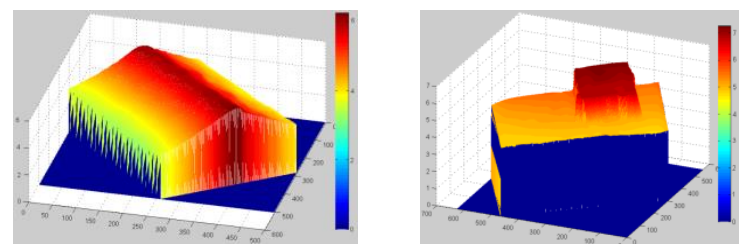

(a)
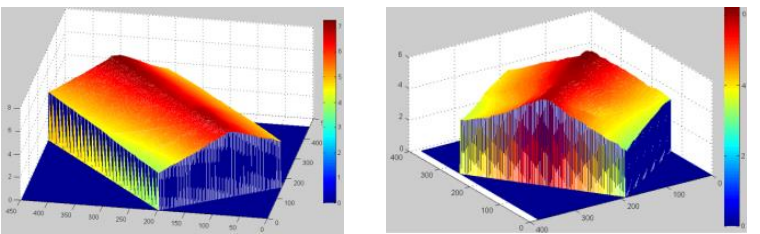

(b)
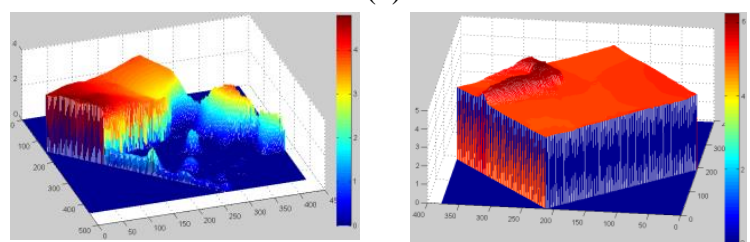

(c)
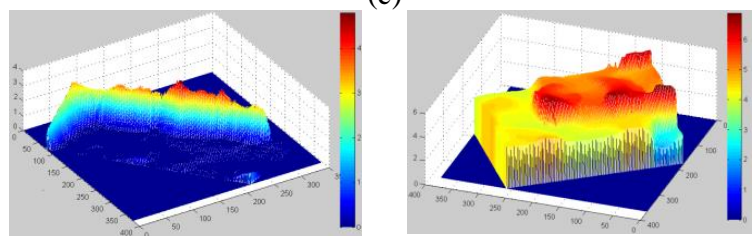

(d)

Figure 2. 3D models for four types of building damage classification: (a) basically intact buildings; (b) slightly damage buildings; (c) partially collapsed buildings; (d) totally collapsed buildings.

In general, heavy damage grades such as collapsed category can be detected with the satellite imagery. Identification of lower damage grades remains a challenge, even with sub-meter resolution remote sensing data (Dong et al., 2013). As an indispensable supplement for the satellite imagery, the UAV images can make up for the lack of satellite images in a certain extent. On one hand, the UAV imagery has significantly improvement of the spatial resolution to determine the damage for a single building, which can reach to the centimeter. On the other hand, 3D point clouds of the hit areas can be reconstructed based on the higher overlapping from the original UAV images (Lu et al., 2010). In this paper, according to the spectral feature of UAV images and the morphological feature of the reconstructed point clouds, the building damage was classified into four levels: basically intact buildings, slightly damaged buildings, partially collapsed buildings and totally collapsed buildings, and the interpretation rules of damage grades is given. In particular, the slightly damaged buildings are determined using the so-called "roof-holes".

The remainder of this paper is organized as follows. In Section 2 , the proposed method for rural building damage classification from the UAV images is presented in detail. In Section 3, the experimental data and results are described. Finally, the conclusions are drawn in Sections 4.

\section{OUR METHOD}

In this section, we propose a method of rural building damage detected using the UAV images. First, the building damage is 


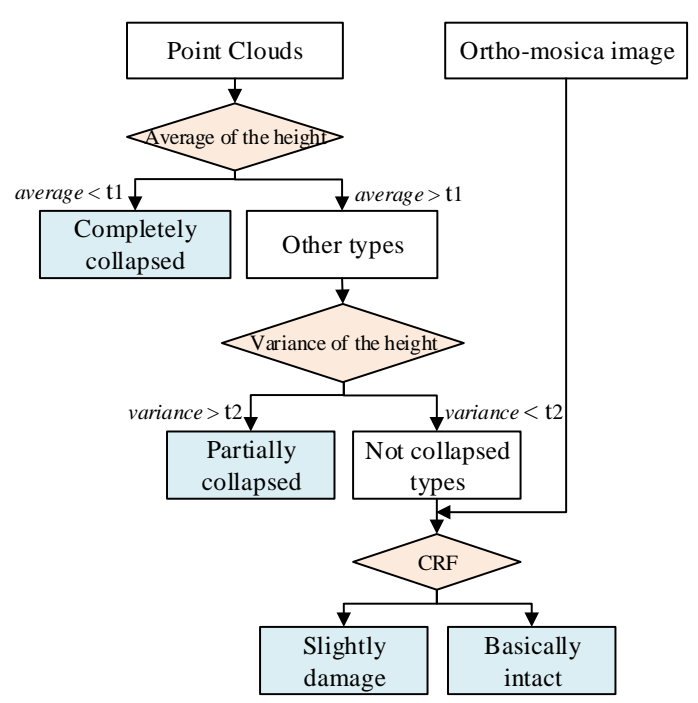

Figure 3. Interpretation rule for building damage types

classified into four levels: basically intact buildings, slightly damaged buildings, partially collapsed buildings and totally collapsed buildings. At the same time, the spectral and morphological features of four types in the UAV images are described. Then, the interpretation rule of building damage classification is given.

\subsection{Building damage classification and image features in the UAV images}

Compared with satellite images, UAV images have two obvious advantages: First, it has higher spatial resolution, which has rich spatial details, clear geometric feature and texture. Second, UAV images have higher overlapping. 3D point cloud data can be reconstructed based on the higher overlapping from the original UAV images. It is exciting that point cloud data contains the morphological features of buildings, such as the height of buildings.

According to spectral feature of UAV images and morphological feature of the reconstructed point cloud data, combined with the existing building damage classification standards, the building damage in the UAV images is classified into four levels: basically intact buildings, slightly damaged buildings, partially collapsed buildings and totally collapsed buildings. The spectral feature and morphological feature of four types are described as follows:

2.1.1 Basically intact buildings: Spectral features: the roof of the buildings is intact, and the distribution of brightness is clear and the hue is regular. The texture of the roof is clear and arranged in order, as shown in Figure 1(a).

Morphological features: the buildings have clear boundaries and geometric shapes, and no rubble was accumulated around the buildings. The 3D point cloud of the buildings is complete, and the average height of buildings in the point clouds approximates the height of the common buildings. 3D models of the intact buildings are shown in Figure 2(a).

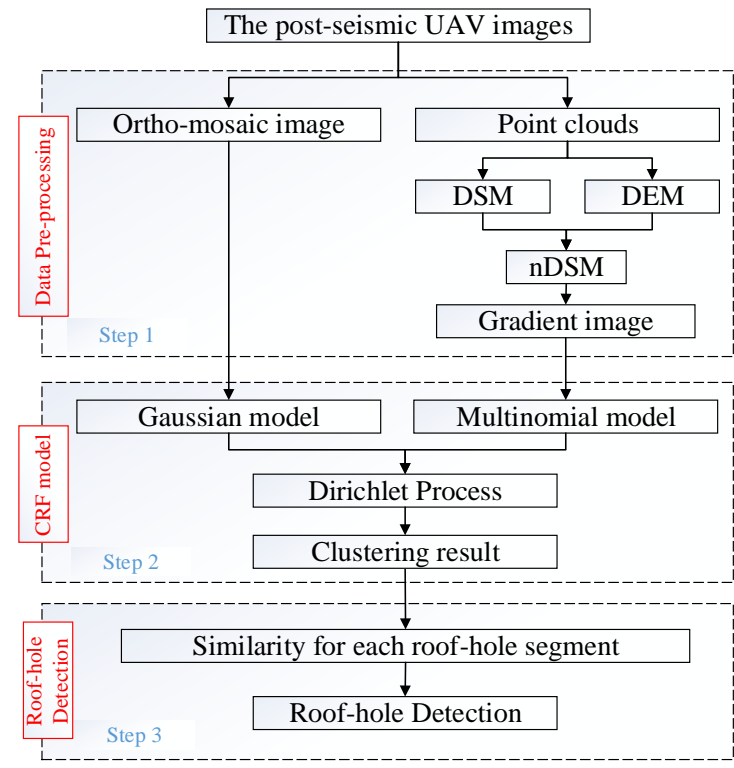

Figure 4. Flowchart of the proposed method for detecting roof-holes

2.1.2 Slightly damaged buildings: In fact, there are many types of slightly damaged buildings, such as there are cracks in the walls, wall skin falls off, and tiles fall from the roofs, etc., and these types can not be detected from remote sensing images. Fortunately, the case of tiles fell from the roofs is the most easily detected using the satellite images. Therefore, slightly damaged buildings refer to the case of tiles fell from the roofs in this paper, which is called "roof-hole", as shown in Figure 1(b).

Spectral features: most of the roof is intact, and the distribution of brightness has well homogeneity. However, some of the tiles fell from the roof, and the color of the roof-holes becomes dark, and the texture is arranged in disorder.

Morphological features: the geometric boundary of the building is clear. Since only a few tiles fell off, the average height of the buildings is similar to the basically intact buildings, and the $3 \mathrm{D}$ model is approximatively completed, as shown in Figure 2(b). However, the height in the roof-holes is slightly lower than its surroundings in the $3 \mathrm{D}$ point clouds.

2.1.3 Partially collapsed buildings: Spectral features: for the partially collapsed buildings, there are collapsed part and not-collapsed part. The collapsed part of the roofs shows black and messy spots in the images, and the regularity of the texture in the collapsed area is destroyed. For the not-collapsed part of the roofs, the spectral feature is similar to the basically intact buildings.

Morphological features: the boundary of the buildings disappears, and the buildings do not have totally geometric shapes and linear texture. The roof of the buildings is partially collapsed. In the 3D point clouds, the average height of notcollapsed part of the buildings is still similar to the basically intact buildings. However, the average height of collapsed part is much lower than that of not-collapsed part, as shown in Figure 2(c). Therefore, the variance of the height of the buildings is larger than that of the basically intact buildings. 


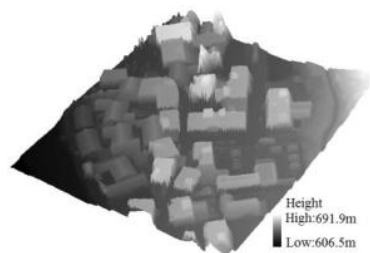

(a)

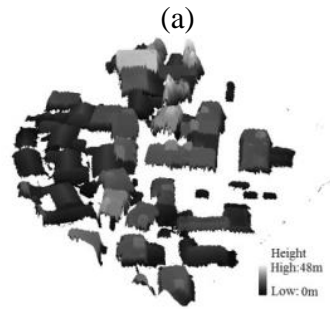

(c)

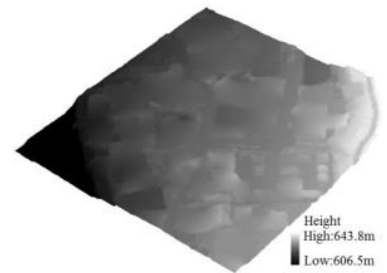

(b)

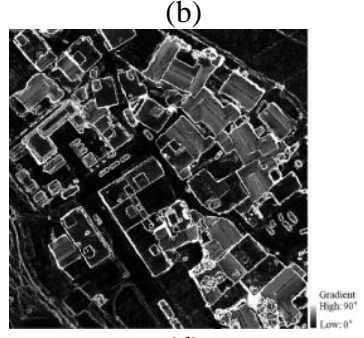

(d)
Figure 5. Pix4UAV derived data: (a) DSM; (b) DEM; (c) nDSM; (d) gradient image.

2.1.4 Totally collapsed buildings: Spectral features: The totally collapsed buildings show black and messy spots in the images. The regularity of the texture is destroyed. Spectral feature of the totally collapsed buildings is similar to the collapsed part of the partially collapsed buildings.

Morphological features: the boundary of the buildings disappears, and the buildings do not have totally geometric shapes. The roof of the buildings is totally collapsed, and a lot of rubble is accumulated around the buildings. In the $3 \mathrm{D}$ point clouds, the average height of buildings is much lower than that of the basically intact buildings, as shown in Figure 2(d).

\subsection{Interpretation rules of building damage classification}

In order to interpret the building damage classification, the first step is to find the most obvious image feature that distinguishes some building damage classification from others. The characteristics of the buildings in the image are color, texture, geometric shape and height. Since the uncertainty of image interpretation and the limitation of image processing, the interpretation of building damage classification using remote sensing images mainly depends on manually visual interpretation. In this paper, according to spectral feature of the UAV images and the morphological feature of point cloud data, the interpretation rule of building damage classification with UAV images is proposed, as shown in Figure 3.

The UAV images have very high resolution, clear texture and rich spatial details, which are obvious advantages for visual interpretation. However, the UAV images have some limitation: due to the abundance of spatial details in the UAV images, the buildings often have complex spectral information, and it lead to the phenomenon of "the same object with different spectrums". Therefore, the result is not satisfactory if the building damage classification is identified only based on the spectral information of the UAV images. Fortunately, 3D point cloud data reconstructed by the UAV images can complement the drawback. Since the height of the buildings is a significant feature in determining the building damage classification, the 3D point clouds of the buildings are taken as the main basis to judge the building damage classification.

Before determining the building damage classification, we assume that vector boundary of each building in the study area

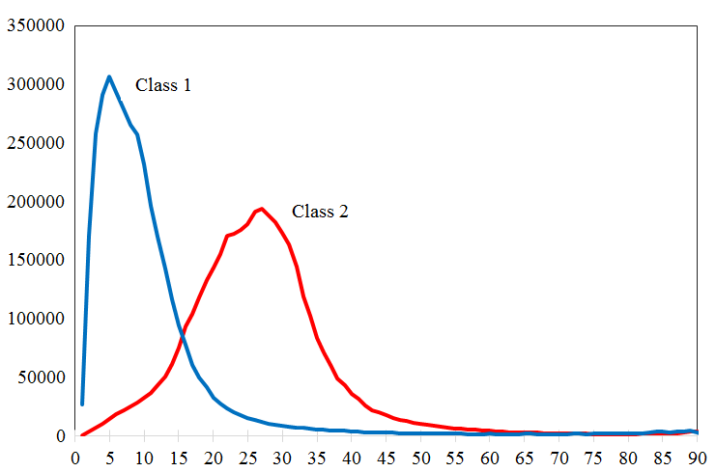

Figure 6. Histogram of two classes of the gradient image

is known, and the point clouds of each building is extracted according to vector boundary of the buildings. Then, 3D model of each building is reconstructed in order to calculate the mean and variance of the height for each building. The specific approach is given as follows:

The threshold $t_{1}$ is set for the average of the height of the building. If the average of the height is less than $t_{1}$, the building is determined to be totally collapsed. Otherwise, it belongs to other types.

Among other types, the threshold $t_{2}$ is set for the variance of the height. If the variance of the height is greater than $t_{2}$, the building is determined to be partially collapsed. Otherwise, it belongs to not-collapsed types.

For the non-collapsed buildings, it is difficult to distinguish between slightly damaged buildings and basically intact buildings only based on the average and variances of the heights. In order to detect the slightly damaged buildings, an unsupervised method of roof-holes detection using joint color and shape features is proposed, which will be described in detail in the next. The slightly damaged buildings can be determined using the detected roof-holes in the roof. The detected roofholes overlap the vector boundary of the buildings, if the interior of the building boundary contains the roof-holes, the building can be taken as slightly damaged building; Otherwise, it belongs to basically intact building.

\subsection{Roof-hole detection based on Chinese restaurant franchise model}

In this section, the Chinese restaurant franchise (CRF) model is employed to detect roof-holes with the post-seismic UAV images. The flowchart of the proposed method is shown in Figure 4, which consists of three sequent steps: (1) the orthomosaic image and gradient image of the study area are derived from the UAV images; (2) the two images are fused using the CRF model to obtain a clustering result; (3) the roof-holes are detected from this clustering result. More details on roof-hole detection can be found in the paper of $\mathrm{Li}$ et al.

2.3.1 Data pre-processing: The post-seismic UAV images are first processed using the Pix4UAV software (Strecha et al., 2011). After that the ortho-mosaic image and 3D point clouds of the study area are derived.

In order to employ the shape feature of point clouds, they need to be further processed. Firstly, the original point clouds were transferred to DSM [Figure 5(a)] in ENVI IDL and filtered to 


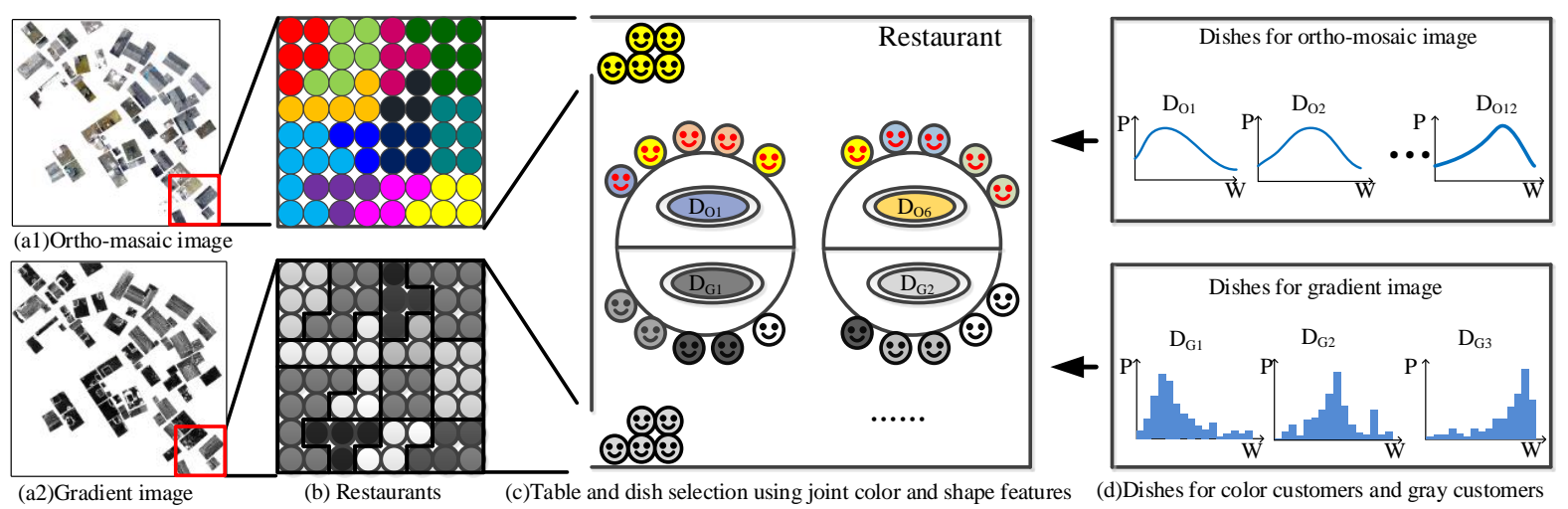

Figure 7. An illustration of the roof-hole detection based on the CRF model.

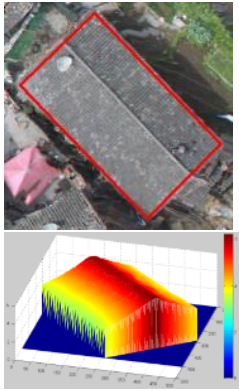

(a)

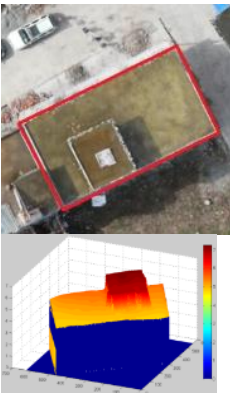

(b)

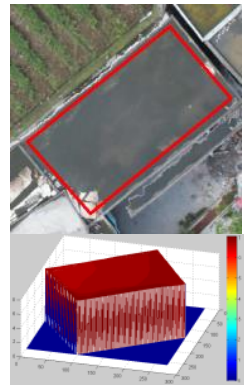

(c)
Figure 8. Types of buildings in Ya'an earthquake and

Wenchuan earthquake: (a) herringbone type; (b) convex type; (c) rectangular type.

extracted terrain XYZ points using morphological filter. The terrain points were further processed in ArcMap to generate DEM [Figure 5(b)], and both DSM and DEM were resampled to the same resolution of ortho-mosaic image. Then, the normalized DSM (nDSM) [Figure 5(c)] was generated by subtracting the DEM from the DSM, which only represents the non-terrain objects. The primary advantage of the nDSM is that it removes the effect of undulation of topography in the mountain area. Finally, a gradient image was generated from the nDSM using the algorithm (Burrough et al., 1998).

According to the ortho-mosaic and gradient images, the roofholes have two obvious characteristics: Firstly, for color feature, the roof-holes get darker than its neighbors of the whole building. Secondly, for shape feature, the roof-holes emerged in the building with pitched roofs in the gradient image. As shown in Figure 6, in Class 1, most pixels have lower gradient value, which indicates the buildings with flat roofs. In contrast, the gradient in Class 2 is slightly higher than that of Class 1, which indicates the buildings with pitched roofs. Since most of roofholes occurred in the pitched roofs, the pixels in Class 2 consist of the "roof-holes". Next, we will discuss how to detect the roof-holes using the joint color and shape features with the CRF model.
2.3.2 The Chinese restaurant franchise with joint color and shape features: The CRF model, a construction method on the hierarchical Dirichlet process (HDP), is firstly applied in information retrieval and text modeling. In the CRF metaphor, suppose that a customer enters a restaurant, the customer randomly choose to either sit at an existing table or start a new table; customers who sat at a table randomly select a dish to eat. More details on table selection and dish selection can be found in the paper of Mao et al. In this paper, in order to describe the clustering problem using UAV images with joint color and shape features, an improved CRF model is proposed to detect the earthquake-triggered roof-holes.

In the proposed model, there are two types of observation data, i.e., an ortho-mosaic image $\boldsymbol{X}^{\boldsymbol{O}}$ with color feature and a gradient image $\boldsymbol{X}^{\boldsymbol{G}}$ with shape feature, of a same geographic location. The two observations are separated into $L$ over-segmentations using the algorithm (Liu et al., 2011). In the CRF model, the "restaurant" is defined as a squared area which contains some neighborhood over-segmentations as shown in Figure 7(b). The "customer $\theta$ " is associated with an over-segmentation, and each customer is described by two feature vectors, i.e., shape and color features. In order to make it clear, we separated the customer $\theta$ with shape and color features into two customers [Figure 7(c)]: one is "color customer $\theta^{O}$ " with color feature, and the other is "gray customer $\theta^{G}$ " with shape feature. The "table $T$ " is the local cluster of the over-segmentation for the orthomosaic and gradient images. The "dish $\boldsymbol{D}^{G}$ " is served for "gray

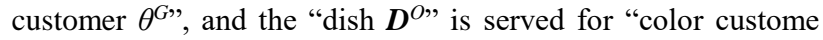
$\theta^{O}$ ", respectively. Thus, the "dish $\boldsymbol{D}^{G O}=\boldsymbol{D}^{G} \times \boldsymbol{D}^{O \text { " }}$ is prepared for the customer $\theta$ with shape and color features. Specifically, the "dishes $\boldsymbol{D}^{G O}$ " are the global clusters of the oversegmentations.

In the proposed method, when a customer $\theta$ with shape and color features enter a restaurant, there exist two sequent random processes, i.e., table selection and dish selection.

Table selection. Assume that all of customers except $\theta_{j}$, have selected tables $T_{\neg j}$ and dishes $D_{\neg j}^{G O}$. The customer $\theta_{j}$ selects a table $t$ using

$$
p\left(t_{j}=t \mid T_{\neg j}, D_{\neg j}^{G O}\right) \propto\left\{\begin{array}{l}
f_{d_{j}^{G O}}^{G}\left(x_{j}^{G}\right) f_{d_{j}^{G O}}^{O}\left(x_{j}^{O}\right), \text { if t has exist } \\
\gamma_{0} f_{d_{j}^{G O}}^{G}\left(x_{j}^{G}\right) f_{d_{j}^{G O}}^{O}\left(x_{j}^{O}\right), \text { if t is a new table }
\end{array}\right.
$$




\begin{tabular}{|c|c|c|}
\hline $\begin{array}{c}\text { Types of } \\
\text { buildings }\end{array}$ & $\begin{array}{c}\text { Building damage } \\
\text { classification }\end{array}$ & $\begin{array}{c}\text { Interpretation rules } \\
(\text { mean }>3 \mathrm{~m})\end{array}$ \\
\hline \multirow{2}{*}{ Herringbone } & Partially collapsed & variance $>2$ \\
\cline { 2 - 3 } Convex & Not-collapsed & variance $<2$ \\
\hline \multirow{2}{*}{ Rectangle } & Partially collapsed & $\begin{array}{c}\text { variance }<2 \text { or } \\
\text { variance }>5\end{array}$ \\
\cline { 2 - 3 } & Not-collapsed & $2<$ variance $<5$ \\
\cline { 2 - 3 } & Portially collapsed & variance $>2$ \\
\hline
\end{tabular}

Table 1. Interpretation rule for partially collapsed buildings in different types of buildings

where $f_{d_{j}^{G O}}^{G}$ and $f_{d_{j}^{G O}}^{O}$ is the likelihood of observations $\boldsymbol{x}^{G}$ and $\boldsymbol{x}^{\boldsymbol{O}}$. $\boldsymbol{x}^{\boldsymbol{G}}$ in the ortho-mosaic image is modelled as a Gaussian distribution, and $x^{o}$ in the gradient image is modelled as a multinomial distribution [Figure $7(\mathrm{~d})] . \gamma_{0}$ is a prior parameter.

If $\boldsymbol{t}$ is a new table, a dish would be selected using

$$
p\left(d_{j^{n e w}}=d \mid T_{\neg j}, D_{\neg j}^{G O}\right) \propto \gamma_{0} f_{d_{j}^{G O}}^{G}\left(x_{j}^{G}\right) f_{d_{j}^{G O}}^{O}\left(x_{j}^{O}\right)
$$

Dish selection. Suppose that all of tables with exception of table $\boldsymbol{t}$ have been served with dishes $D_{\neg j}^{G O}$. A dish $\boldsymbol{d}_{j}$ is selected for the table $t$ using

$$
p\left(d_{j}=d \mid T_{\neg j}, D_{\neg j}^{G O}\right) \propto\left\{\begin{array}{l}
f_{d_{j}^{G O}}^{G}\left(x_{j}^{G}\right) f_{d_{j}^{G O}}^{O}\left(x_{j}^{O}\right), \text { if } d \text { is previously used } \\
\gamma_{0} f_{d_{j}^{G}}^{G}\left(x_{j}^{G}\right) f_{d_{j}^{G}}^{O}\left(x_{j}^{O}\right), \text { if } d \text { is a new dish }
\end{array}\right.
$$

Dish selection is also based on the likelihood of observations $x^{G}$ and $\boldsymbol{x}^{\boldsymbol{O}}$, the same as table selection. After finishing the process of table selection and dish selection, we finally get the clustering result.

2.3.3 Roof-holes detection : After getting the clustering result, the next is to find "roof-hole" segments (i.e., oversegmentations). In this case, it is done by comparing the distribution of each segment in the clustering result and distribution of "roof-hole" segments in the ground truth. The similarity between two distributions is calculated based on Kullback-Leibler (KL) divergence. For each roof-hole segment in the ground truth, all the segments in the clustering result can be sorted according to their scores computed by KL divergence, and the most representative segments with high scores can be selected as detected the roof-holes.

\section{EXPERIMENTS AND DISCUSSIONS}

\subsection{Experimental Data}

In the experiment, the post-earthquake UAV images of Ya'an earthquake and Wenchuan earthquake are used to verify the proposed method. The information of the two earthquakes and the corresponding UAV images are described as follows:

Ya'an earthquake: Ya'an, located at the center of Sichuan, China, experienced an earthquake with a magnitude of 7.0 on April 20, 2013. The first study area, Yuxi village in Ya'an city, is one of the areas most affected by the earthquake. Covered an area of about $0.04 \mathrm{~km}^{2}$, a total of 93 post-earthquake UAV images were obtained on April 24. The average ground sampling distance (GSD) is $2.49 \mathrm{~cm}$.

Wenchuan earthquake: a magnitude 8.0 earthquake hits Wenchuan country in Sichuan province $\left(31.01^{\circ} \mathrm{N}, 103.42^{\circ} \mathrm{E}\right)$ on May 12, 2008. The earthquake caused huge casualties and economic losses. The second study area, Zhoujiawan village in Hanwang town, is in IX degree intensity zone, and it covers an area of about $1.33 \mathrm{~km}^{2}$. A total of $151 \mathrm{UAV}$ images were obtained on May 29, 2009, and the average GSD is $7.91 \mathrm{~cm}$.

The post-earthquake UAV images of the two earthquakes are preprocessed with Pix4UAV commercial software. After that, two types of data are derived: the ortho-mosaic image and 3D point clouds. The point cloud data contains the height information and color information. The average point density of the point clouds in Yuxi village and Zhoujiawan village are 290 points $/ \mathrm{m}^{2}$ and 190 points $/ \mathrm{m}^{2}$, respectively.

In the experiment, the boundaries of the buildings were obtained with manual interpretation from the pre-earthquake VHR satellite images. In order to match the boundaries of the buildings with the post-earthquake UAV images, the preearthquake satellite images and the post-earthquake orthomosaic images were performed with image registration. In the study area of Zhoujiawan village, a total of 320 homonymy points were matched the pre-earthquake QuickBird image with the post-earthquake ortho-mosaic image. Similarly, in the area of Yuxi village, a total of 120 homonymy points were matched the pre-earthquake Pléiades image with the post-earthquake ortho-mosaic image. Since the ranges of two study areas are different, the number of homonymy points is different. For example, the ortho-mosaic image of Zhoujiawan village covers about $1.33 \mathrm{~km}^{2}$, but the residential regions only account for approximately $20 \%$ of the whole image, and most of the homonymy points focus on the residential regions. Therefore, it is believed that it is accurate for the match of pre-earthquake satellite images and the post-earthquake UAV images.

\subsection{Experimental Results and Discussions}

According to geometric shapes of the buildings in the 3D point clouds, the buildings in the two study areas are classified into three types: "herringbone", "convex", and "rectangle", as shown in Figure 9. In the type of "herringbone", the roof of the building shows the obvious strips which cross the whole roof. It is worth noting that the slightly damaged buildings only occur in this type. In the "convex" type, there is no strip in the roofs, and the distribution of brightness is regular. It is interesting that there is a rectangle chimney on the roof, so that the whole building looks like "convex" in the 3D model. In the type of "rectangle", the distribution of brightness of the roof is regular, and it is the most common type in the buildings. Among the three types, there are only the two types of "herringbone" and "rectangle" in the ortho-mosaic image of Zhoujiawan village, and no "convex" type of buildings are found.

After getting the ortho-mosaic images and 3D models of the buildings, the average and variance of each building are counted. According to the interpretation rule of building damage classification given in Section 2.2, the average threshold of the height is firstly set. Since the two study areas located in the rural regions, there exist only one-layer buildings and no multilayer buildings in the two study areas. Therefore, the average of the height is set to 3 meters in the experiments. If the average of 


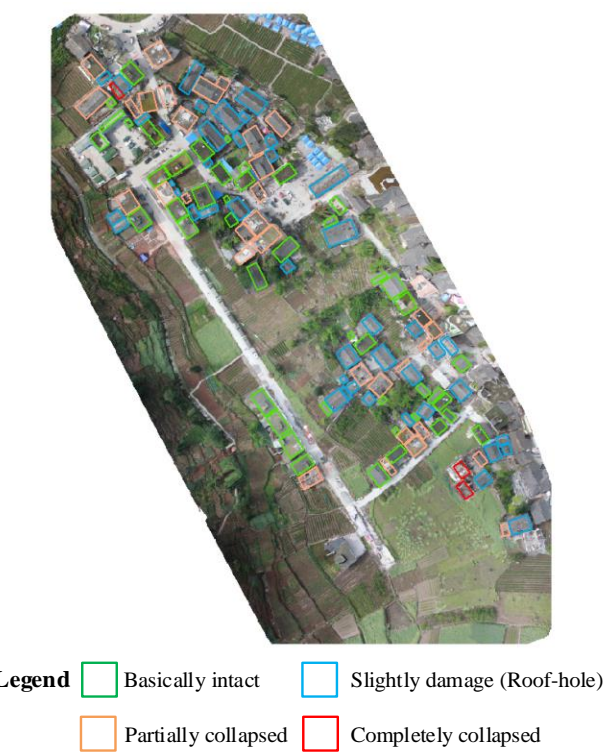

(a)
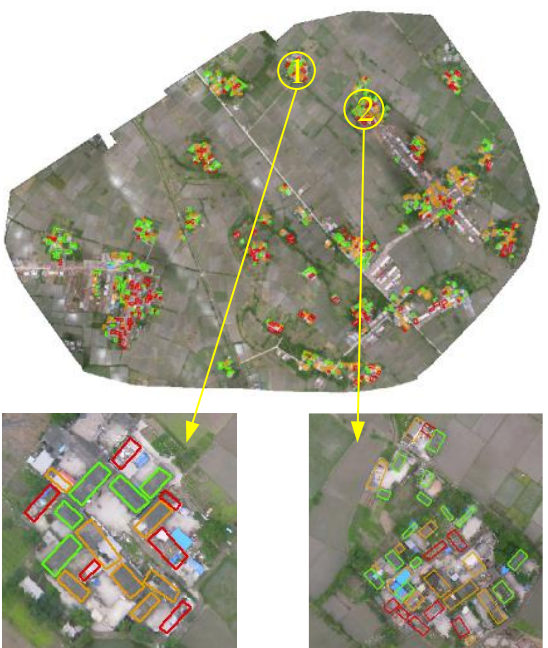

Legend $\square$ Not collapsed $\square$ Partially collapsed $\square$ Completely collapsed

(b)

Figure 9. Results of building damage classification in two study areas: (a) results of building damage in Yuxi village; (b) results of building damage in Zhoujiawan village.

\begin{tabular}{|c|c|c|c|c|}
\hline Study areas & Types of building damage & Ground truth & Correct number & Wrong number \\
\hline \multirow{4}{*}{ Yuxi village } & Basically intact & 46 & 46 & 0 \\
\cline { 2 - 5 } & Slightly damage & 43 & 43 & 0 \\
\cline { 2 - 5 } & Partially collapsed & 33 & 33 & 0 \\
\cline { 2 - 5 } & Totally collapsed & 3 & 3 & 0 \\
\hline \multirow{3}{*}{ Zhoujiawan village } & Not collapsed & 225 & 214 & 11 \\
\cline { 2 - 5 } & Partially collapsed & 179 & 171 & 8 \\
\cline { 2 - 5 } & Totally collapsed & 173 & 164 & 9 \\
\hline
\end{tabular}

Table 2. Quantitative analysis of the results of the building damage classification

the height is less than 3 , the building is determined to be totally collapsed. Otherwise, the building is to be determined.

For the buildings to be determined, variance threshold of the height needs to be set, and the setting of variance is based on the height of $3 \mathrm{~m}$. Due to the different types of buildings, variance thresholds are different, as shown in Table 1. According to the types of the buildings, variance threshold is set as follows:

For the "herringbone" buildings, if the variance is more than 2, the building is determined to be partially collapsed. Otherwise, it belongs to not-collapsed building.

In terms of the "convex" buildings, if the variance is less than 2 or more than 5 , the building is determined to be partially collapsed. Otherwise, it belongs to not-collapsed building. For the "convex" buildings, there exists a certain variance in the basically intact buildings. Therefore, when the variance is less than 2, it implies that the chimney on the roof is destroyed, and it is determined to be partially collapsed. When the variance is more than 5, it implies that part of the roof is collapsed.

For the "rectangle" buildings, there is no obvious difference in variance with the "herringbone" buildings. Therefore, it has the same rules with the "herringbone" buildings. If the variance is more than 2, the building is determined to be partially collapsed. Otherwise, it belongs to not-collapsed building.
In terms of not-collapsed buildings, they are classified into slightly damaged and basically intact buildings. The roof-holes were detected using the CRF model in Section 2.3. The detected roof-holes overlaps the vector boundary of the buildings, if the interior of the building boundary contains the roof-holes, the building can be taken as slightly damaged building; Otherwise, it belongs to basically intact building.

Figure 9 shows the results of building damage classification in Ya'an and Wenchuan earthquakes, respectively. It is noticed that Zhoujiawan village is located in IX degree intensity zone, and the buildings are seriously damaged, and roof-holes on the roofs are not common. Therefore, the building damage classification in Wenchuan earthquake is classified into only three levels: totally collapsed buildings, partially collapsed buildings, and not-collapsed buildings.

The results of building damage classification in the two study areas are quantitatively analyzed in Table 2. For Yuxi village in Ya'an earthquake, there are a total of 125 buildings. They are determined to be 46 basically intact buildings, 43 slightly damaged buildings, 33 partially collapsed buildings, and 3 totally collapsed buildings, which are consistent with the results of visual interpretation.

For Zhoujiawan village of Wenchuan earthquake, there are a total of 581 buildings, and 4 buildings are not determined due 
to the lack of point clouds. Thus only 577 buildings are determined to be 214 non-collapsed buildings, 171 partially collapsed buildings, and 164 totally collapsed buildings. The results of Wenchuan earthquake have some differences with visual interpretation, it is because the UAV images of Zhoujiawan village were shot a year after Wenchuan earthquake, and several totally collapsed buildings were replaced by new temporary buildings. It leads to the fact that some totally collapsed buildings are misjudged as not-collapsed buildings. In addition, there exist some new buildings built on vacant lands, such as the external walls of buildings. But the vector boundaries of buildings are obtained from the pre-earthquake satellite images. Therefore, the new buildings shown in the post-earthquake UAV image have no boundary.

In a word, the results of building damage classification in the two study areas are consistent with that of visual interpretation, and the results verify the effectiveness of the proposed method.

\section{CONCLUSIONS}

In this paper, according to the post-earthquake UAV images and the reconstructed point clouds, the building damage in the UAV images is classified into four levels: basically intact, slightly damaged, partially collapsed and totally collapsed buildings. At the same time, the spectral and morphological features of four types are described, and interpretation rule of building damage classification is given. Specially, an unsupervised method was presented to detect earthquake-induced roof-holes using UAV images with joint color and shape features, and the slightly damaged buildings are determined using the detected roof-holes. In the experiment, the post-earthquake UAV images of Ya'an and Wenchuan earthquakes are used to verify the proposed method.

\section{ACKNOWLEDGEMENTS}

This work was partly supported by National key R\&D program of China (No. 2017YFB0504104) and the Doctoral Foundation of Hebei Normal University (No. L2018B19).

\section{REFERENCES}

Burrough, P.A., and McDonell, R.A., 1998. Principles of Geographical Information Systems, Oxford University Press, New York, pp. 190.

Chesnel, A.L., Binet, R., and Wald, L., 2007. Object oriented assessment of damage due tonatural disaster using very high resolution images. In: IEEE International Geoscience and Remote Sensing Symposium (IGARSS), pp. 3736-3739.

Dong, L., and Shan, J., 2013. A comprehensive review of earthquake-induced building damage detection with remote sensing techniques. ISPRS Journal of Photogrammetry and Remote Sensing, 84, pp. 85-99.

Gamba, P., Acqua, F.D., and Trianni, G., 2007. Rapid damage detection in Bam area using multitemporal SAR and exploiting ancillary data. IEEE Transactions on Geoscience and Remote Sensing, 45(6), 1582-1589.

Gamba, P., Dell Acqua, F., and Odasso, L., 2007. Objectoriented building damage analysis in VHR optical satellite images of the 2004 tsunami over Kalutara, Sri Lanka. In: Joint Urban Remote Sensing Event (JURSE), Paris, France.
Grunthal, G., 1998. European macroseismic scale 1998. Cahiers du Centre Europeen de Geodynamique et de Seismologie, 15, pp. 1-99.

Gusella, L., Adams, B.J., Bitelli, G.C., Huyck, K., Mognol, A., 2005. Object-oriented image understanding and post-earthquake damage assessment for the 2003 Bam, Iran, earthquake. Earthquake Spectra, 21(S1), pp. 225-238.

Iwasaki, Y., and Yamazaki, F., 2011. Detection of building collapse from the shadow lengths in optical satellite images. In: 32nd Asian Conference on Remote Sensing. Taiwan, China.

Schweier, C., and Markus, M., 2006. Classification of collapsed buildings for fast damage and loss assessment. Bulletin of Earthquake Engineering, 4, pp. 177-192.

Saito, K., Spence, R.J., Going, C., and Markus, M., 2004. Using high-resolution satellite images for post-earthquake building damage assessment: a study following the 26 January 2001 Gujarat earthquake. Earthquake Spectra, 20(1), pp. 145-170.

Li, P.J., Xu, H.Q., and Guo, J.C., 2009. Urban building damage detection from very high resolution imagery using OCSVM and spatial features. International Journal of Remote Sensing, 31(13), pp. 3393-3409.

Li, S.D., Tang, H., He, S., Shu, Y., Mao, T., Li, J., and Xu, Z.H., 2015. Unsupervised detection of earthquake-triggered roofholes from UAV images using joint color and shape features. IEEE Geoscience and Remote Sensing Letters, 12(9), pp. 18231827.

Liu, J.H., Shan, X.J., and Yin, J.Y., 2004. Automatic recognition of damaged town buildings caused by earthquake using remote sensing information: Taking the 2001 BHUJ, India Earthquake and the 1976 Tangshan, China Earthquake as examples. Acta Seismologica Sinica, 26(6), pp. 623-632.

Liu, M.Y., Tuzel, O., Ramalingam, S., and Chellappa, R., 2011. Entropy Rate Superpixel Segmentation. In: IEEE Computer Vision and Pattern Recognition (CVPR), USA.

Lu, H., Li, Y.S., He, J., Chen, Q., and Ren, Z.P., 2010. An automatic mosaic method in unmanned aerial vehicle images based on feature points. Geography and Geo-Information Science, 26(5), pp. 16-19.

Mao, T., Tang, H., Wu, J.J., Jiang, W.G, He, S., and Shu, Y., 2016. A generalized metaphor of Chinese restaurant franchise to fusing both panchromatic and multispectral images for unsupervised classification. IEEE Transactions on Geoscience and Remote Sensing, 54(8): pp. 4594-4604.

Strecha, C., and Küng, O., 2011. Pix4UAV Software, Open Source Geospatial Foundation http://www.pix4d.com.

Tong, X.H., Hong, Z.H., and Liu, S.J., 2012. Building-damage detection using pre- and post-seismic high-resolution satellite stereo imagery: a case study of May 2008 Wenchuan earthquake. ISPRS Journal of Photogrammetry and Remote Sensing, 68(2), pp. 13-27.

Yamazaki, F., Vu, T.T., and Matsuoka, M., 2007. Contextbased detection of post-disaster damaged buildings in urban areas from satellite images. In: Urban Remote Sensing Joint Event (JURSE), Paris, France. 